\title{
Sharp constants for inequalities of Poincaré type: an application of optimal control theory
}

Hongwei Lou*

\section{"Correspondence:}

hwlou@fudan.edu.cn

School of Mathematical Sciences,

and LMNS, Fudan University,

Shanghai, 200433, China

\begin{abstract}
Sharp constants for an inequality of Poincaré type are studied. The problem is solved by using optimal control theory.
\end{abstract}

MSC: 26D10; 46E35; 49K15

Keywords: sharp constant; inequality of Poincaré type; optimal control

\section{Introduction}

Denote by $W^{1,2}(-1,1)$ the Sobolev space of all real-valued functions $f(\cdot)$ that are absolutely continuous on the closed interval $[-1,1]$ and such that $f^{\prime}(\cdot) \in L^{2}(-1,1)$. Let $m \geq 1$ be an integer. Denote by $W(1,2, m)$ the space

$$
W(1 ; 2, m) \triangleq\left\{y(\cdot) \in W^{1,2}(-1,1) \mid \int_{-1}^{1} t^{k} y(t) d t=0(0 \leq k \leq m-1)\right\} .
$$

Kalyabin considered in [1] the following problem.

Problem $\left(\boldsymbol{B}_{x}\right)$ Fix $x \in[-1,1]$, find the best constant $B_{m}(x)$ such that the following inequality holds:

$$
|y(x)| \leq B_{m}(x)\left(\int_{-1}^{1}\left|y^{\prime}(t)\right|^{2} d t\right)^{\frac{1}{2}}, \quad \forall y(\cdot) \in W(1,2, m) .
$$

It is proved in [1] that

$$
B_{m}^{2}( \pm 1)=\frac{2}{m(m+2)}, \quad B_{1}^{2}(x)=\frac{1+3 x^{2}}{6}, \quad B_{2}^{2}(0)=\frac{1}{6}
$$

and the extremal functions for the case $x=1$ are

$$
C\left((m+2) P_{m}(t)+m P_{m+1}(t)\right)
$$

where $C$ is a constant and

$$
P_{k}(t)=\frac{1}{2^{k} k !} \frac{d^{k}}{d t^{k}}\left(t^{2}-1\right)^{k}, \quad k=0,1,2, \ldots,
$$


are the classical Legendre polynomials. For notational simplicity, we denote

$$
p_{k}(t)=\frac{1}{2^{k} k !} \frac{d^{k-2}}{d t^{k-2}}\left(t^{2}-1\right)^{k}, \quad k=2,3, \ldots
$$

and

$$
q_{k}(x)=\frac{\int_{-1}^{1} P_{k}(s)|s-x| d s}{\int_{-1}^{1}\left|P_{k}(s)\right|^{2} d s}= \begin{cases}\frac{x^{2}+1}{2}, & \text { if } k=0 \\ \frac{x^{3}-3 x}{2}, & \text { if } k=1, \\ (2 k+1) p_{k}(x), & \text { if } k \geq 2\end{cases}
$$

Many problems similar to Problem $\left(B_{x}\right)$ were studied; see, for example, [2, 3], and [4]. In this paper, we will solve Problem $\left(B_{x}\right)$ completely with the help of optimal control theory. Since the cases $x= \pm 1$ were solved in [1], we mainly consider the cases of $x \in(-1,1)$. We have the following.

Theorem 1.1 Assume $m \geq 1$ and $x \in(-1,1)$. Then, $y(\cdot) \in W(1,2, m)$ is an extremal function to Problem $\left(B_{x}\right)$ if and only if

$$
y(t)=C\left[c(x)\left(Q_{m+1}(t)-|t-x|\right)+1\right], \quad t \in[-1,1],
$$

where $C$ is a constant,

$$
\begin{aligned}
& Q_{m}(t)=A(x)+\sum_{1 \leq k \leq m-1} q_{k}(x) P_{k}(t)+\alpha(x) P_{m}(t)+\beta(x) P_{m+1}(t), \quad t \in[-1,1], \\
& c(x)=\frac{2}{x^{2}+1-2 A(x)},
\end{aligned}
$$

and $A(x), \alpha(x), \beta(x)$ are characterized by

$$
Q_{m}^{\prime}(-1)=-1, \quad Q_{m}^{\prime}(1)=1
$$

and

$$
Q_{m}(x)=0 .
$$

The sharp constant of the inequality (1.2) is

$$
B_{m}(x)=\frac{1}{\sqrt{2 c(x)}}, \quad x \in(-1,1)
$$

More precisely, we have the following.

Corollary 1.2 Assume $m=1$ and $x \in(-1,1)$. Then

$$
B_{1}(x)=\sqrt{\frac{3 x^{2}+1}{6}}
$$


and $y(\cdot) \in W(1,2,1)$ is an extremal function to Problem $\left(B_{x}\right)$ if and only if

$$
y(t)=C\left[\frac{3}{3 x^{2}+1}\left(\frac{t^{2}-x^{2}}{2}-|t-x|\right)+1\right], \quad t \in[-1,1]
$$

Corollary 1.3 Assume $m=2$ and $x \in(-1,1)$. Then

$$
B_{2}(x)=\sqrt{\frac{8-21 x^{2}+30 x^{4}-5 x^{6}}{48}}
$$

and $y(\cdot) \in W(1,2,2)$ is an extremal function to Problem $\left(B_{x}\right)$ if and only if

$$
y(t)=C\left[\frac{4+33 x^{2}-30 x^{4}+5 x^{6}+8 P_{2}(t)-2\left(x^{3}-3 x\right)\left(P_{3}(t)-6 P_{1}(t)\right)-24|t-x|}{8-21 x^{2}+30 x^{4}-5 x^{6}}+1\right] .
$$

Corollary 1.4 Assume $m=2 n+1, n \geq 1$ and $x \in(-1,1)$. Then

$$
\begin{aligned}
B_{2 n+1}(x)= & \left\{\frac{\left(x^{2}-1\right)^{2}}{4}+\frac{1}{2} \sum_{k=2}^{2 n}(2 k+1) p_{k}(x) P_{k}(x)\right. \\
& -\frac{1}{2(n+1)(2 n+1)}\left[\frac{x^{3}-3 x}{2}+\sum_{1 \leq k \leq n-1}(k+1)(2 k+1)(4 k+3) p_{2 k+1}(x)\right] P_{2 n+1}(x) \\
& \left.+\frac{1}{2(n+1)(2 n+3)}\left[1-\sum_{k=1}^{n} k(2 k+1)(4 k+1) p_{2 k}(x)\right] P_{2 n+2}(x)\right\}^{\frac{1}{2}} .
\end{aligned}
$$

In particular,

$$
\begin{aligned}
& B_{3}(x)=\frac{\sqrt{297+1,260 x^{2}-5,370 x^{4}+5,900 x^{6}-1,575 x^{8}}}{16 \sqrt{15}}, \\
& B_{5}(x)=\frac{\sqrt{1,375+8,400 x^{2}-95,025 x^{4}+357,560 x^{6}-597,555 x^{8}+448,056 x^{10}-121,275 x^{12}}}{16 \sqrt{105}} .
\end{aligned}
$$

Corollary 1.5 Assume $m=2 n+2, n \geq 1$ and $x \in(-1,1)$. Then

$$
\begin{aligned}
B_{2 n+2}(x)= & \left\{\frac{\left(x^{2}-1\right)^{2}}{4}+\frac{1}{2} \sum_{k=2}^{2 n+1}(2 k+1) p_{k}(x) P_{k}(x)\right. \\
& +\frac{1}{2(n+1)(2 n+3)}\left[1-\sum_{k=1}^{n} k(2 k+1)(4 k+1) p_{2 k}(x)\right] P_{2 n+2}(x) \\
& -\frac{1}{2(n+2)(2 n+3)}\left[\frac{x^{3}-3 x}{2}+\sum_{k=1}^{n}(k+1)(2 k+1)(4 k+3) p_{2 k+1}(x)\right] \\
& \left.\times P_{2 n+3}(x)\right\}^{\frac{1}{2}} .
\end{aligned}
$$


In particular,

$$
\begin{aligned}
B_{4}(x)= & \frac{\sqrt{297-1,440 x^{2}+9,030 x^{4}-20,860 x^{6}+18,585 x^{8}-5,292 x^{10}}}{16 \sqrt{15}}, \\
B_{6}(x)= & \frac{1}{32 \sqrt{42}}\left(2,200-15,225 x^{2}+211,050 x^{4}-1,162,455 x^{6}+3,017,700 x^{8}\right. \\
& \left.-3,977,127 x^{10}+2,562,714 x^{12}-637,065 x^{14}\right)^{\frac{1}{2}} .
\end{aligned}
$$

When $x=0$, we have the following.

Corollary 1.6 The following holds:

$$
B_{1}^{2}(0)=B_{2}^{2}(0)=\frac{1}{6}, \quad B_{3}^{2}(0)=B_{4}^{2}(0)=\frac{99}{1,280}
$$

and

$$
\begin{aligned}
B_{2 n+1}^{2}(0)= & B_{2 n+2}^{2}(0)=\frac{3}{32}-\sum_{k=2}^{n} \frac{(4 k+1)}{2^{4 k+2}(k+1)(2 k-1)}\left(\begin{array}{c}
2 k \\
k
\end{array}\right)^{2} \\
& +(-1)^{n} \frac{\left(\begin{array}{c}
2 n+2 \\
n+1
\end{array}\right)}{2^{2 n+2}\left(\begin{array}{c}
2 n+3 \\
2
\end{array}\right)}\left[\frac{7}{16}-\sum_{k=2}^{n} \frac{(-1)^{k} k(4 k+1)}{2^{2 k+3}(2 k-1)}\left(\begin{array}{c}
2 k+2 \\
k+1
\end{array}\right)\right], \quad n \geq 2 .
\end{aligned}
$$

In particular,

$$
B_{5}^{2}(0)=B_{6}^{2}(0)=\frac{275}{5,376}, \quad B_{7}^{2}(0)=B_{8}^{2}(0)=\frac{45,325}{1,179,648} .
$$

\section{Transmitting Problem $\left(B_{x}\right)$ to optimal control problem}

We introduce the equivalent optimal control problem to Problem $\left(B_{x}\right)$. Let $U=L^{2}(-1,1)$. We define the following control system:

$$
\frac{d}{d t}\left(\begin{array}{c}
y(t) \\
w(t) \\
z_{0}(t) \\
z_{1}(t) \\
\vdots \\
z_{m-1}(t)
\end{array}\right)=\left(\begin{array}{c}
u(t) \\
u(t) \chi_{(-1, x)}(t) \\
y(t) \\
t y(t) \\
\vdots \\
t^{m-1} y(t)
\end{array}\right), \quad t \in(-1,1)
$$

and the state constraints

$$
y(-1)=w(-1), \quad w(1)=1, \quad z_{k}( \pm 1)=0 \quad(k=0,1, \ldots, m-1) .
$$

Let

$$
\mathcal{P}_{\text {ad }}=\left\{(Y(\cdot), u(\cdot)) \in\left(W^{1,2}(-1,1)\right)^{m+2} \times \mathcal{U}(Y(\cdot), u(\cdot)) \text { satisfying }(2.1)-(2.2)\right\}
$$


and

$$
\mathcal{U}_{a d}=\left\{u(\cdot) \mid(Y(\cdot), u(\cdot)) \in \mathcal{P}_{a d}\right\}
$$

where

$$
Y(\cdot)=\left(\begin{array}{c}
y(\cdot) \\
w(\cdot) \\
z_{0}(\cdot) \\
z_{1}(\cdot) \\
\vdots \\
z_{m-1}(\cdot)
\end{array}\right) .
$$

Our optimal control problem corresponding to Problem $\left(B_{x}\right)$ is as follows.

Problem $\left(C_{x}\right)$ Let $x \in(-1,1)$. Find $(\bar{Y}(\cdot), \bar{u}(\cdot)) \in \mathcal{P}_{a d}$ such that

$$
\int_{-1}^{1} \bar{u}^{2}(t) d t=\inf _{(Y(\cdot), u(\cdot)) \in \mathcal{P}_{a d}} \int_{-1}^{1} u^{2}(t) d t .
$$

It is obvious that $(Y(\cdot), u(\cdot)) \mapsto y(\cdot)$ is a bijection from $\mathcal{P}_{a d}$ to $\{f(\cdot) \in W(1,2, m) \mid f(x)=1\}$. Then one can easily see that

$$
B_{m}(x)=\left(\inf _{(Y(\cdot), u(\cdot)) \in \mathcal{P}_{a d}} \int_{-1}^{1} u^{2}(t) d t\right)^{-\frac{1}{2}}
$$

Therefore, we can solve Problem $\left(B_{x}\right)$ by solving Problem $\left(C_{x}\right)$.

\section{Pontryagin's maximum principle}

We state Pontryagin's maximum principle for optimal control problems. Symbols in this section will have similar but probably different meanings from other sections. Thus we set this part as a separate section. We will state a result given in [5]. For simplicity, we only state it in a simple way. In other words, Lemma 3.1 below is a special case of Theorem 3.1 and Corollary 3.1 in Chapter V of [5].

Now, let $T>t_{0}$ and $U \subseteq \mathbb{R}^{m}$. A measurable function $u(\cdot)$ defined on $\left[t_{0}, T\right]$ with range in $U$ is said to be a control.

Let the function $\hat{f}=\left(f^{0}, f\right)=\left(f^{0}, f^{1}, \ldots, f^{n}\right)$ be an $\mathbb{R}^{n+1}$-valued vector function on $\left[t_{0}, T\right] \times$ $\mathbb{R}^{n} \times \mathbb{R}^{m}$. Assume that $\hat{f}$ is Borel measurable on $t \in\left[t_{0}, T\right]$, continuous on $(y, u) \in \mathbb{R}^{n} \times \mathbb{R}^{m}$ and continuously differentiable on $y \in \mathbb{R}^{n}$.

If $y(\cdot)$ is an absolutely function on $\left[t_{0}, T\right]$ with range in $\mathbb{R}^{n}$ such that

$$
\frac{d y(t)}{d t}=f(t, y(t), u(t)), \quad \text { a.e. on }\left[t_{0}, T\right]
$$

then $y(\cdot)$ is called a state/trajectory corresponding to $u(\cdot)$.

Let $\Omega \subseteq \mathbb{R}^{n} \times \mathbb{R}^{n}$ be a $C^{1}$ manifold of dimensional $k$, where $0 \leq k \leq 2 n$. We say $(y(\cdot), u(\cdot))$ is an admissible pair if

(i) $u(\cdot)$ is a control, 
(ii) $y(\cdot)$ is a state corresponding to $u(\cdot)$,

(iii) $\left(y\left(t_{0}\right), y(T)\right) \in \Omega$.

Denote by $\mathcal{P}_{a d}$ the set of all admissible pairs. The set $u_{a d}=\left\{u(\cdot) \mid(Y(\cdot), u(\cdot)) \in \mathcal{P}_{a d}\right\}$ is called the set of admissible controls.

If an admissible pair $(\bar{y}(\cdot), \bar{u}(\cdot))$ satisfies

$$
J(\bar{y}(\cdot), \bar{u}(\cdot))=\inf _{(y(\cdot), u(\cdot)) \in \mathcal{P}_{a d}} J(y(\cdot), u(\cdot)),
$$

then it is called an optimal pair, where

$$
J(y(\cdot), u(\cdot)):=\int_{0}^{T} f^{0}(t, y(t), u(t)) d t .
$$

Assume that for each compact $Y \subset \mathbb{R}^{n}$ and admissible control $u(\cdot)$, there exists a function $\mu(\cdot)=\mu(\cdot ; Y, u(\cdot))$ such that for almost all $t \in\left[t_{0}, T\right]$ and all $y \in Y$,

$$
|\hat{f}(t, y, u(t))| \leq \mu(t), \quad\left|\hat{f}_{y}(t, y, u(t))\right| \leq \mu(t) .
$$

We have the following.

Lemma 3.1 Let assumptions listed in this section hold. Let $(\bar{y}(\cdot), \bar{u}(\cdot))$ be an optimal pair. Then there exists a constant $\lambda^{0} \leq 0$ and an absolutely continuous vector function $\lambda=$ $\left(\lambda_{1}, \lambda_{2}, \ldots, \lambda_{n}\right)$ defined on $\left[t_{0}, T\right]$ such that the following hold:

(i) The vector $\left(\lambda^{0}, \lambda(t)\right)$ is never zero on $\left[t_{0}, T\right]$.

(ii) For a.e. $t \in\left[t_{0}, T\right]$,

$$
\left.\lambda^{\prime}(t)=-f_{y}(t, \bar{y}(t), \bar{u}(t)) \lambda(t)-\lambda^{0} f_{y}^{0} t, \bar{y}(t), \bar{u}(t)\right)
$$

where

$$
f_{y}=\left(\begin{array}{cccc}
\frac{\partial f_{1}}{\partial y_{1}} & \frac{\partial f_{2}}{\partial y_{1}} & \cdots & \frac{\partial f_{n}}{\partial y_{1}} \\
\frac{\partial f_{1}}{\partial y_{2}} & \frac{\partial f_{2}}{\partial y_{2}} & \cdots & \frac{\partial f_{n}}{\partial y_{2}} \\
\vdots & \vdots & \ddots & \vdots \\
\frac{\partial f_{1}}{\partial y_{n}} & \frac{\partial f_{2}}{\partial y_{n}} & \cdots & \frac{\partial f_{n}}{\partial y_{n}}
\end{array}\right) .
$$

(iii) The pointwise maximum condition holds: for almost all $t \in\left[t_{0}, T\right]$ and all $u \in U$,

$$
\lambda^{0} f^{0}(t, \bar{y}(t), \bar{u}(t))+\langle\lambda(t), f(t, \bar{y}(t), \bar{u}(t))\rangle \geq \lambda^{0} f^{0}(t, \bar{y}(t), \bar{u}(t))+\langle\lambda(t), f(t, \bar{y}(t), u)\rangle .
$$

(iv) The transversality condition holds: if the mapping $t \mapsto \hat{f}(t, \bar{y}(t), \bar{u}(t))$ is continuous at $t=t_{0}$ and $t=T$, then $\left(-\lambda\left(t_{0}\right), \lambda(T)\right)$ is orthogonal to $\Omega$.

\section{Proof of Theorem 1.1}

We give the following lemma first.

Lemma 4.1 Let $n \geq 1, c \in \mathbb{R}, x \in(-1,1), Q(\cdot)$ is an $(n+1)$ th degree polynomial satisfying

$$
Q(x)=0, \quad Q^{\prime}(1)=1, \quad Q^{\prime}(-1)=-1
$$


and

$$
\int_{-1}^{1} t^{k}[c(Q(t)-|t-x|)+1] d t=0, \quad \forall k=0,1, \ldots, n-1
$$

Then

$$
\int_{-1}^{1}\left[\frac{\partial}{\partial t}(Q(t)-|t-x|)\right]^{2} d t=\frac{2}{c}
$$

Proof Noting that $Q^{\prime \prime}(\cdot)$ is an $(n-1)$ th degree polynomial, by $(4.2)$, we have

$$
\int_{-1}^{1} Q^{\prime \prime}(t)[c(Q(t)-|t-x|)+1] d t=0 .
$$

Therefore,

$$
\begin{aligned}
\int_{-1}^{1} & {\left[\frac{\partial}{\partial t}(Q(t)-|t-x|)\right]^{2} d t } \\
& =\int_{-1}^{x}\left(Q^{\prime}(t)+1\right)^{2} d t+\int_{x}^{1}\left(Q^{\prime}(t)-1\right)^{2} d t \\
& =\int_{-1}^{1}\left(Q^{\prime}(t)\right)^{2} d t+2+2 \int_{-1}^{x} Q^{\prime}(t) d t-2 \int_{x}^{1} Q^{\prime}(t) d t \\
& =Q(1) Q^{\prime}(1)-Q(-1) Q^{\prime}(-1)-\int_{-1}^{1} Q^{\prime \prime}(t) Q(t) d t+2+4 Q(x)-2 Q(-1)-2 Q(1) \\
& =\int_{-1}^{1} Q^{\prime \prime}(t)\left(\frac{1}{c}-|t-x|\right) d t+2-Q(-1)-Q(1) \\
& =\frac{2}{c}+\int_{-1}^{x} Q^{\prime \prime}(t)(t-x) d t+\int_{x}^{1} Q^{\prime \prime}(t)(x-t) d t+2-Q(-1)-Q(1) \\
& =\frac{2}{c}-(1+x)-\int_{-1}^{x} Q^{\prime}(t) d t-(1-x)+\int_{x}^{1} Q^{\prime}(t) d t+2-Q(-1)-Q(1) \\
= & \frac{2}{c} .
\end{aligned}
$$

Now, we list some properties of Legendre polynomials. We can easily get

$$
\begin{aligned}
& P_{k}(0)= \begin{cases}0, & \text { if } k=2 n+1, n \geq 0, \\
1, & \text { if } k=0, \\
(-1)^{n} \frac{(2 n-1) ! !}{(2 n) ! !}, & \text { if } k=2 n, n \geq 1,\end{cases} \\
& p_{k}(0)= \begin{cases}0, & \text { if } k=2 n+1, n \geq 1, \\
\frac{1}{8}, & \text { if } k=2, \\
(-1)^{n-1} \frac{(2 n-3) ! !}{(2 n+2) ! !} & \text { if } k=2 n, n \geq 2,\end{cases} \\
& P_{k}(-1)=(-1)^{k}, \quad P_{k}(1)=1, \\
& P_{k}^{\prime}(-1)=(-1)^{k-1} \frac{k(k+1)}{2}, \quad P_{k}^{\prime}(1)=\frac{k(k+1)}{2},
\end{aligned}
$$




$$
\int_{-1}^{1}\left|P_{k}(t)\right|^{2} d t=\frac{2}{2 k+1}
$$

and

$$
\begin{aligned}
& \int_{-1}^{1}\left(P_{k}^{\prime}(t)\right)^{2} d t=P_{k}^{\prime}(1) P_{k}(1)-P_{k}^{\prime}(-1) P_{k}(-1)=k(k+1), \\
& \int_{-1}^{1} P_{k}^{\prime}(t) P_{k+1}^{\prime}(t) d t=P_{k}^{\prime}(1) P_{k+1}(1)-P_{k}^{\prime}(-1) P_{k+1}(-1)=0, \\
& \int_{-1}^{1} P_{k}(t)|t-x| d t= \begin{cases}x^{2}+1, & \text { if } k=0, \\
\frac{1}{3} x^{3}-x, & \text { if } k=1, \\
2 p_{k}(x), & \text { if } k \geq 2 . \\
\end{cases}
\end{aligned}
$$

We turn to the proof of Theorem 1.1.

\section{Proof of Theorem 1.1}

I. Existence of optimal pair. One can prove directly that the sharp constant $B_{m}(x)$ is attainable, i.e., there is a nontrivial $\bar{y}(\cdot) \in W(1,2, m)$ such that

$$
|\bar{y}(x)|=B_{m}(x)\left(\int_{-1}^{1}\left|y^{\prime}(t)\right|^{2} d t\right)^{-\frac{1}{2}} .
$$

Now, we give an optimal control version of this fact.

Let $\left(Y_{j}(\cdot), u_{j}(\cdot)\right) \in \mathcal{P}_{a d}$ be a minimizing sequence of Problem $\left(C_{x}\right)$. That is

$$
\lim _{j \rightarrow+\infty} \int_{-1}^{1} u_{j}^{2}(t) d t=\inf _{(Y(\cdot), u(\cdot)) \in \mathcal{P}_{a d}} \int_{-1}^{1} u^{2}(t) d t .
$$

Then $u_{j}(\cdot)$ is bounded in $L^{2}(-1,1)$. That is

$$
\left\|u_{j}(\cdot)\right\|_{L^{2}(-1,1)} \leq M
$$

for some constant $M>0$. Denote $Y_{j}(\cdot) \equiv\left(y^{j}(\cdot), w^{j}(\cdot), z_{0}^{j}(\cdot), \ldots, z_{m-1}^{j}(\cdot)\right)$. Then, by the state equation (2.1) and the constraints (2.2), we have

$$
\int_{-1}^{1} y^{j}(t) d t=z_{0}^{j}(1)-z_{0}^{j}(-1)=0 .
$$

Then (2.1)-(2.2), and Poincaré's inequality imply

$$
\left\|y^{j}(\cdot)\right\|_{W^{1,2}(-1,1)} \leq C_{1}, \quad\left\|w^{j}(\cdot)\right\|_{W^{1,2}(-1,1)} \leq C_{1}, \quad \forall j \geq 1
$$

and consequently

$$
\left\|z_{k}^{j}(\cdot)\right\|_{W^{1,2}(-1,1)} \leq C_{2}, \quad \forall k=0,1, \ldots, m-1 ; j \geq 1
$$

for some constant $C_{1}, C_{2}$. That is, $Y_{j}(\cdot)$ is bounded in $\left(W^{1,2}(-1,1)\right)^{m+2}$. Then, by Sobolev's imbedding theorem, $Y_{j}(\cdot)$ is bounded and equicontinuous in $(C[-1,1])^{m+2}$. 
Thus, Eberlein-Shmulyan's theorem and Arzelá-Ascoli's theorem (see Chapter V, Appendix 4 and Chapter III, Section 3 in [6], for example), we can suppose that

$$
u_{j}(\cdot) \rightarrow \bar{u}(\cdot), \quad \text { weakly in } L^{2}(-1,1)
$$

and

$$
Y_{j}(\cdot) \rightarrow \bar{Y}(\cdot), \quad \text { weakly in }\left(W^{1,2}(-1,1)\right)^{m+2} \text {, strongly in }(C[-1,1])^{m+2}
$$

for some $\bar{u}(\cdot) \in L^{2}(-1,1)$ and $\bar{Y}(\cdot) \in\left(W^{1,2}(-1,1)\right)^{m+2}$. One can easily see that $(\bar{Y}(\cdot), \bar{u}(\cdot))$ satisfies (2.1) and (2.2). Thus, $(\bar{Y}(\cdot), \bar{u}(\cdot)) \in \mathcal{P}_{\text {ad }}$. Moreover,

$$
\int_{-1}^{1} \bar{u}^{2}(t) d t \leq \lim _{j \rightarrow+\infty} \int_{-1}^{1} u_{j}^{2}(t) d t
$$

Therefore $(\bar{Y}(\cdot), \bar{u}(\cdot))$ is a solution to Problem $\left(C_{x}\right)$. We call it an optimal pair of Problem $\left(C_{x}\right)$.

II. Pontryagin's maximum principle for the optimal pair. We now apply Lemma 4.1 Pontryagin's maximum principle to Problem $\left(C_{x}\right)$. We can easily verify that all the conditions posed in Section 3 hold. For example, conditions on state constraints and the local existence of a dominating integrable function (see (3.1)) hold. More precisely, let

$$
\Omega=\{(s, s) \mid s \in \mathbb{R}\} \times\{0\}^{m} \times \mathbb{R} \times\{1\} \times\{0\}^{m} .
$$

Then $\Omega$ is a $C^{1}$ manifold of dimensional 2. While the state constraints (2.2) is equivalent to $(Y(-1), Y(1)) \in \Omega$.

On the other hand, for any $u(\cdot) \in L^{2}(-1,1)$ and $|Y| \leq R$, if we choose $\mu(t)=2|u(t)|+m R$, then the condition (4.1) corresponding to (2.1) holds.

Now, by Lemma 4.1, the optimal pair $(\bar{Y}(\cdot), \bar{u}(\cdot))$ satisfies the following Pontryagin maximum principle: there exists a $\varphi^{0} \leq 0$ and a solution to the following adjoint equation:

$$
\frac{d}{d t}\left(\begin{array}{c}
\varphi(t) \\
\zeta(t) \\
\psi_{0}(t) \\
\psi_{1}(t) \\
\vdots \\
\psi_{m-1}(t)
\end{array}\right)=\left(\begin{array}{c}
-\sum_{j=0}^{m-1} t^{j} \psi_{j}(t) \\
0 \\
0 \\
0 \\
\vdots \\
0
\end{array}\right), \quad t \in(-1,1)
$$

such that the following conditions hold:

(i) we have the following non-trivial condition:

$$
\left(\varphi^{0}, \varphi(\cdot), \zeta(\cdot), \psi_{0}(\cdot), \ldots, \psi_{m-1}(\cdot)\right) \neq 0
$$

(ii) the maximum condition:

$$
\begin{aligned}
\varphi^{0} & \bar{u}^{2}(t)+\left(\varphi(t)+\zeta(t) \chi_{(-1, x)}(t)\right) \bar{u}(t) \\
& =\max _{u \in \mathbb{R}}\left[\varphi^{0} u^{2}+\left(\varphi(t)+\zeta(t) \chi_{(-1, x)}(t)\right) u\right], \quad \text { a.e. } t \in(-1,1),
\end{aligned}
$$


(iii) the transversality condition

$$
\varphi(-1)+\zeta(-1)=0, \quad \varphi(1)=0 .
$$

III. Simplification. By $(4.21), \zeta(\cdot) \equiv \zeta, \psi_{0}(\cdot) \equiv \psi_{0}, \ldots, \psi_{m-1}(\cdot) \equiv \psi_{m-1}$ are constants and

$$
\varphi^{\prime}(t)=-\sum_{j=0}^{m-1} \psi_{j} t^{j}, \quad t \in(-1,1) .
$$

Then $\varphi(\cdot)$ is an $m$ th degree polynomial.

By (4.23), we have

$$
2 \varphi^{0} \bar{u}(t)+\varphi(t)+\zeta \chi_{(-1, x)}=0, \quad \text { a.e. } t \in(-1,1) .
$$

If $\varphi^{0}=0$, we get

$$
\varphi(t)=\left\{\begin{array}{ll}
-\zeta, & t \in(-1, x), \\
0, & t \in[x, 1),
\end{array} \text { a.e. } t \in(-1,1)\right.
$$

Therefore, since $\varphi(\cdot)$ is a polynomial, we have the following:

$$
\varphi(\cdot) \equiv 0, \quad \zeta=0 .
$$

Consequently, by (4.25),

$$
\psi_{0}=\psi_{1}=\cdots=\psi_{m-1}=0 .
$$

This contradicts the non-trivial condition (4.22). Therefore, we must have $\varphi^{0}<0$. Without loss of generality, we can suppose that $\varphi^{0}=-\frac{1}{2}$. Then it follows from (4.26) that

$$
\bar{u}(t)=\varphi(t)+\zeta \chi_{(-1, x)}(t), \quad t \in(-1,1) .
$$

Combining the above with (4.24), we see that the corresponding function $\bar{y}(\cdot)$ is continuously differentiable on $[-1, x) \cup(x, 1]$ and

$$
\bar{y}^{\prime}( \pm 1)=\bar{u}( \pm 1)=0 .
$$

Moreover, $\bar{y}(\cdot)$ can be expressed as

$$
\bar{y}(t)=-c(x)|t-x|+\widetilde{Q}_{m}(t), \quad t \in[-1,1],
$$

where $c \equiv c(x)=\frac{\zeta}{2}$ and $\widetilde{Q}_{m}(\cdot)$ is an $(m+1)$ th degree polynomial. 
We claim that $c \neq 0$. Otherwise, $c=0$. Then it follows from (4.31) and

$$
\int_{-1}^{1} t^{k} \bar{y}(t) d t=0, \quad k=0,1,2, \ldots, m-1
$$

that

$$
\widetilde{Q}_{m}(t)=c_{m} P_{m}(t)+c_{m+1} P_{m+1}(t), \quad k=0,1,2, \ldots, m-1
$$

for some constant $c_{m}, c_{m+1}$.

Then (4.30) and (4.9) imply $c_{m}=c_{m+1}=0$. This contradicts the nontrivial condition. Therefore $c \neq 0$ and we can rewrite $\bar{y}(\cdot)$ as

$$
\bar{y}(t)=c(x)\left(Q_{m}(t)-|t-x|\right)+1, \quad t \in[-1,1],
$$

where $Q_{m}(\cdot)$ is an $(m+1)$ th degree polynomial such that

$$
Q_{m}(x)=0, \quad Q_{m}^{\prime}(-1)=-1, \quad Q_{m}^{\prime}(1)=1
$$

IV. Conclusion. By (4.32),

$$
\int_{-1}^{1} Q_{m}(t) P_{k}(t) d t=\int_{-1}^{1} P_{k}(t)|x-t| d t, \quad 1 \leq k \leq m-1 .
$$

Thus we see that

$$
Q_{m}(t)=A(x)+\sum_{1 \leq k \leq m-1} q_{k}(x) P_{k}(t)+\alpha(x) P_{m}(t)+\beta(x) P_{m+1}(t)
$$

where $q_{k}(x)$ is defined by (1.7). Moreover, by (4.35), we can determine $A(x), \alpha(x)$ and $\beta(x)$. Finally, using (4.32) again, we get

$$
c(x)=\frac{2}{x^{2}+1-2 A(x)} .
$$

By Lemma 4.1, we have the following:

$$
\int_{-1}^{1}|\bar{u}(t)|^{2} d t=\int_{-1}^{1}\left|\bar{y}^{\prime}(t)\right|^{2} d t=2 c(x)
$$

Then Theorem 1.1 follows from (2.7).

Remark 4.1 If $x=-1$, instead of (4.34)-(4.35), we get

$$
\bar{y}(t)=Q_{m}(t), \quad t \in[-1,1]
$$

with

$$
Q_{m}(-1)=1, \quad Q_{m}^{\prime}(1)=0
$$


and

$$
Q_{m}(t)=\alpha P_{m}(t)+\beta P_{m+1}(t), \quad t \in[-1,1] .
$$

The above equations imply the results in [1] for $x= \pm 1$.

On the other hand, since $B_{m}(x)$ is obviously continuous respect to $x \in[-1,1]$, we can certainly get $B_{m}( \pm 1)$ from Theorem 1.1 .

\section{Results for some special cases}

We prove Corollaries 1.2-1.6 in this section.

Proof of Corollary 1.2 By (1.9), we have

$$
Q_{1}(t)=A(x)+\alpha(x) P_{1}(t)+\beta(x) P_{2}(t) .
$$

Thus (1.11) and (4.9) imply $\alpha(x)=0, \beta(x)=\frac{1}{3}$. Then (1.12) implies

$$
A(x)=-\frac{1}{3} P_{2}(x)=\frac{1-3 x^{2}}{6} .
$$

Consequently,

$$
Q_{1}(t)=\frac{1}{3}\left(P_{2}(t)-P_{2}(x)\right)=\frac{t^{2}-x^{2}}{2}
$$

and

$$
c(x) \equiv \frac{2}{x^{2}+1-2 A(x)}=\frac{3}{3 x^{2}+1}
$$

Therefore, the extremal functions to Problem $\left(B_{x}\right)$ are $C \bar{y}(\cdot)$ with

$$
\bar{y}(x)=\frac{3}{3 x^{2}+1}\left(\frac{t^{2}-x^{2}}{2}-|t-x|\right)+1,
$$

while

$$
B_{1}(x)=\frac{1}{\sqrt{2 c(x)}}=\sqrt{\frac{3 x^{2}+1}{6}} .
$$

Proof of Corollary 1.3 By (1.7) and (1.9), we have

$$
Q_{2}(t)=A(x)+\frac{x^{3}-3 x}{2} P_{1}(t)+\alpha(x) P_{2}(t)+\beta(x) P_{3}(t) .
$$

Then it follows easily from (1.11) that

$$
\alpha(x)=\frac{1}{3}, \quad \beta(x)=-\frac{x^{3}-3 x}{12} .
$$


Then, by (1.12),

$$
A(x)=\frac{4+33 x^{2}-30 x^{4}+5 x^{6}}{24} .
$$

Thus

$$
c(x)=\frac{2}{x^{2}+1-2 A(x)}=\frac{24}{8-21 x^{2}+30 x^{4}-5 x^{6}} .
$$

Therefore

$$
B_{2}(x)=\frac{1}{\sqrt{2 c(x)}}=\sqrt{\frac{8-21 x^{2}+30 x^{4}-5 x^{6}}{48}}
$$

and the extremal functions to Problem $\left(B_{x}\right)$ are

$$
C\left(\frac{4+33 x^{2}-30 x^{4}+5 x^{6}+8 P_{2}(t)-2\left(x^{3}-3 x\right)\left(P_{3}(t)-6 P_{1}(t)\right)-24|t-x|}{8-21 x^{2}+30 x^{4}-5 x^{6}}+1\right) .
$$

Proof of Corollary 1.4 By (1.7) and (1.9), we have

$$
\begin{aligned}
Q_{2 n+1}(t)= & A(x)+\frac{x^{3}-3 x}{2} P_{1}(t)+\sum_{k=2}^{2 n}(2 k+1) p_{k}(x) P_{k}(t) \\
& +\alpha(x) P_{2 n+1}(t)+\beta(x) P_{2 n+2}(t) .
\end{aligned}
$$

Then by (1.11)-(1.12), and (1.10),

$$
\begin{aligned}
\alpha(x)= & -\frac{1}{(n+1)(2 n+1)}\left[\frac{x^{3}-3 x}{2}+\sum_{1 \leq k \leq n-1}(k+1)(2 k+1)(4 k+3) p_{2 k+1}(x)\right] \\
\beta(x)= & \frac{1}{(n+1)(2 n+3)}\left[1-\sum_{k=1}^{n} k(2 k+1)(4 k+1) p_{2 k}(x)\right] \\
A(x)= & -\frac{x^{3}-3 x}{2} P_{1}(x)-\sum_{k=2}^{2 n}(2 k+1) p_{k}(x) P_{k}(x) \\
& +\frac{1}{(n+1)(2 n+1)}\left[\frac{x^{3}-3 x}{2}+\sum_{1 \leq k \leq n-1}(k+1)(2 k+1)(4 k+3) p_{2 k+1}(x)\right] P_{2 n+1}(x) \\
& -\frac{1}{(n+1)(2 n+3)}\left[1-\sum_{k=1}^{n} k(2 k+1)(4 k+1) p_{2 k}(x)\right] P_{2 n+2}(x) \\
\frac{1}{2 c(x)}= & \frac{x^{2}+1}{4}-\frac{A(x)}{2}=\frac{\left(x^{2}-1\right)^{2}}{4}+\frac{1}{2} \sum_{k=2}^{2 n}(2 k+1) p_{k}(x) P_{k}(x) \\
& -\frac{1}{2(n+1)(2 n+1)}\left[\frac{x^{3}-3 x}{2}+\sum_{1 \leq k \leq n-1}(k+1)(2 k+1)(4 k+3) p_{2 k+1}(x)\right] P_{2 n+1}(x) \\
& {\left[1-\sum_{k=1}^{n} k(2 k+1)(4 k+1) p_{2 k}(x)\right] P_{2 n+2}(x) }
\end{aligned}
$$

Finally, (1.19) and (1.20) follow from direct calculations. We get the proof. 
Proof of Corollary 1.5 By (1.7) and (1.9), we have

$$
\begin{aligned}
Q_{2 n+2}(t)= & A(x)+\frac{x^{3}-3 x}{2} P_{1}(t)+\sum_{k=2}^{2 n+1}(2 k+1) p_{k}(x) P_{k}(t) \\
& +\alpha(x) P_{2 n+2}(t)+\beta(x) P_{2 n+3}(t) .
\end{aligned}
$$

Then by (1.11)-(1.12), and (1.10),

$$
\begin{aligned}
& \alpha(x)=\frac{1}{(n+1)(2 n+3)}\left[1-\sum_{k=1}^{n} k(2 k+1)(4 k+1) p_{2 k}(x)\right], \\
& \beta(x)=-\frac{1}{(n+2)(2 n+3)}\left[\frac{x^{3}-3 x}{2}+\sum_{k=1}^{n}(k+1)(2 k+1)(4 k+3) p_{2 k+1}(x)\right] \text {, } \\
& A(x)=-\frac{x^{3}-3 x}{2} P_{1}(x)-\sum_{k=2}^{2 n+1}(2 k+1) p_{k}(x) P_{k}(x) \\
& -\frac{1}{(n+1)(2 n+3)}\left[1-\sum_{k=1}^{n} k(2 k+1)(4 k+1) p_{2 k}(x)\right] P_{2 n+2}(x) \\
& +\frac{1}{(n+2)(2 n+3)}\left[\frac{x^{3}-3 x}{2}+\sum_{k=1}^{n}(k+1)(2 k+1)(4 k+3) p_{2 k+1}(x)\right] \\
& \times P_{2 n+3}(x) \text {, } \\
& \frac{1}{2 c(x)}=\frac{x^{2}+1}{4}-\frac{A(x)}{2} \\
& =\frac{\left(x^{2}-1\right)^{2}}{4}+\frac{1}{2} \sum_{k=2}^{2 n+1}(2 k+1) p_{k}(x) P_{k}(x) \\
& +\frac{1}{2(n+1)(2 n+3)}\left[1-\sum_{k=1}^{n} k(2 k+1)(4 k+1) p_{2 k}(x)\right] P_{2 n+2}(x) \\
& -\frac{1}{2(n+2)(2 n+3)}\left[\frac{x^{3}-3 x}{2}+\sum_{k=1}^{n}(k+1)(2 k+1)(4 k+3) p_{2 k+1}(x)\right] \\
& \times P_{2 n+3}(x) .
\end{aligned}
$$

Finally, (1.21) and (1.22) follow from direct calculations. We get the proof.

Proof of Corollary 1.6 First, we get (1.24) from (1.14), (1.16), (1.19), and (1.22).

By $(4.7), p_{2 k+1}(0)=0(k=1,2, \ldots)$. Thus, if $n \geq 2$, we get from (1.18) and (1.21)

$$
\begin{aligned}
B_{2 n+1}^{2}(0)= & B_{2 n+2}^{2}(0) \\
= & \frac{1}{4}+\frac{1}{2} \sum_{k=1}^{n}(4 k+1) p_{2 k}(0) P_{2 k}(0) \\
& +\frac{1}{2(n+1)(2 n+3)}\left[1-\sum_{k=1}^{n} k(2 k+1)(4 k+1) p_{2 k}(0)\right] P_{2 n+2}(0) .
\end{aligned}
$$


Moreover, using (4.6)-(4.7), we get (1.25):

$$
\begin{aligned}
B_{2 n+1}^{2}(0)= & B_{2 n+2}^{2}(0) \\
= & \frac{1}{4}+\frac{1}{2} \times \frac{5}{8} \times\left(-\frac{1}{2}\right)-\frac{1}{2} \sum_{k=2}^{n}(4 k+1) \frac{(2 k-3) ! !}{(2 k+2) ! !} \frac{(2 k-1) ! !}{(2 k) ! !} \\
& -\frac{(-1)^{n}}{2(n+1)(2 n+3)}\left[1-\frac{15}{8}+\sum_{k=2}^{n}(-1)^{k} k(2 k+1)(4 k+1) \frac{(2 k-3) ! !}{(2 k+2) ! !}\right] \frac{(2 n+1) ! !}{(2 n+2) ! !} \\
= & \frac{3}{32}-\sum_{k=2}^{n} \frac{(4 k+1)}{2^{4 k+2}(k+1)(2 k-1)}\left(\begin{array}{c}
2 k \\
k
\end{array}\right)^{2} \\
& +(-1)^{n} \frac{\left(\begin{array}{c}
2 n+2 \\
2+1
\end{array}\right)}{2^{2 n+2}\left(\begin{array}{c}
2 n+3 \\
2
\end{array}\right)}\left[\frac{7}{16}-\sum_{k=2}^{n} \frac{(-1)^{k} k(4 k+1)}{2^{2 k+3}(2 k-1)}\left(\begin{array}{c}
2 k+2 \\
k+1
\end{array}\right)\right], \quad n \geq 2 .
\end{aligned}
$$

Now (1.26) follows directly from (1.25).

\section{Competing interests}

The author declares that they have no competing interests.

\section{Acknowledgements}

This work was supported in part by 973 Program (No. 2011CB808002) and NSFC (No. 11371104).

Received: 24 December 2013 Accepted: 23 April 2014 Published: 29 December 2014

\section{References}

1. Kalyabin, GA: Sharp constants in one-dimensional inequalities of Poincaré type. Math. Notes 90(4), 634-636 (2011) (in Russian). Translation in Math. Notes, 90(3-4), 615-618 (2011)

2. Gerasimov, IV, Nazarov, Al: Best constant in a three-parameter Poincaré inequality. J. Math. Sci. 179(1), 80-99 (2011)

3. Oshime, $Y$, Watanabe, $\mathrm{K}$ : The best constant of $L^{p}$ Sobolev inequality corresponding to Dirichlet boundary value problem II. Tokyo J. Math. 34(1), 115-133 (2011)

4. Talenti, G: Best constant in Sobolev inequality. Ann. Mat. Pura Appl. (4) 110, 353-372 (1976)

5. Berkovitz, LD: Optimal Control Theory. Springer, New York (1983)

6. Yosida, K: Functional Analysis, 6th edn. Springer, Berlin (1980)

\section{Submit your manuscript to a SpringerOpen ${ }^{\circ}$ journal and benefit from:}

- Convenient online submission

Rigorous peer review

- Immediate publication on acceptance

- Open access: articles freely available online

- High visibility within the field

- Retaining the copyright to your article 